\title{
Case report of isochromosome $17 q$ in acute myeloid leukemia with myelodysplasia-related changes after treatment with a hypomethylating agent
}

\author{
J.C. Sousa ${ }^{1}$, R.T. Germano ${ }^{2}$, C.C.M. Castro ${ }^{3}$, S.M.M. Magalhaes ${ }^{1}$ and \\ R.F. Pinheiro ${ }^{1,3}$ \\ ${ }^{1}$ Programa de Pós-Graduação em Ciências Médicas, \\ Departamento de Medicina, Universidade Federal do Ceará, \\ Fortaleza, CE, Brasil \\ ${ }^{2}$ Universidade Estadual do Ceará, Fortaleza, CE, Brasil \\ ${ }^{3}$ Departamento de Patologia, Universidade Federal do Ceará, \\ Fortaleza, CE, Brasil \\ Corresponding author: R.F. Pinheiro \\ E-mail: ronaldpinheiro@pq.cnpq.br
}

Genet. Mol. Res. 11 (3): 2045-2050 (2012)

Received November 8, 2011

Accepted March 27, 2012

Published August 6, 2012

DOI http://dx.doi.org/10.4238/2012.August.6.8

ABSTRACT. Isochromosome 17q is a relatively common karyotypic
abnormality in medulloblastoma, gastric, bladder, and breast cancers.
In myeloid disorders, it is observed during disease progression and
evolution to acute myeloid leukemia in Philadelphia-positive chronic
myeloid leukemia. It has been reported in rare cases of myelodysplastic
syndrome, with an incidence of $0.4-1.57 \%$. Two new agents have
been approved for treatment of myelodysplastic syndrome/chronic
myelomonocytic leukemia. These are the hypomethylating agents,
5-azacytidine and decitabine, recommended by consensus guidelines
for high-risk myelodysplastic syndrome patients not eligible for 
hematopoietic stem cell transplantation. We present a case of chronic myelomonocytic leukemia with normal cytogenetics at diagnosis treated with decitabine (with good response); however, the patient evolved to acute myeloid leukemia with $i(17 q)$ shortly after suspending treatment. To the best of our knowledge, this is the first report of acute myeloid leukemia with myelodysplasia-related changes with $\mathrm{i}(17 \mathrm{q})$ after the use of a hypomethylating agent.

Key words: Myelodysplastic syndromes; Isochromosome 17q; Hypomethylation therapy

\section{INTRODUCTION}

Isochromosome $17 \mathrm{q}[\mathrm{i}(17 \mathrm{q})]$ is a relatively common karyotypic abnormality in medulloblastoma and gastric, bladder, and breast cancers. In myeloid disorders, it is observed during disease progression and evolution to acute myeloid leukemia (AML) in Philadelphiapositive chronic myeloid leukemia (Mitelman, 1995). It has been reported in rare cases of myelodysplastic syndrome (MDS), with an incidence of $0.4-1.57 \%$ (Marisavljevic et al., 2004). The appropriate categorization of hematological neoplasms associated with isolated $\mathrm{i}(17 \mathrm{q})$ is uncertain. A proportion of cases would be more appropriately categorized as MDS/myeloproliferative neoplasm (MPN), unclassified.

Defined by the World Health Organization in 2008, AML with myelodysplasia-related changes is a new category of AML that includes a previous myelodysplastic phase or MDS/myeloproliferative phase (Brunning et al., 2008). Chronic myelomonocytic leukemia (CMML) is a clonal hematopoietic neoplasia characterized by features of both MPN and MDS (Brunning et al., 2008).

In 2006, we reported an 81-year-old man with MDS (refractory anemia with excess blasts II) who displayed a bone marrow G-banded karyotype: 46,XY,del(7)(q32),i(17)(q10). This report was the first of $\mathrm{i}(17 \mathrm{q})$ plus the deletion of $7 \mathrm{q}$ in MDS. The patient became dependent on red blood cell (RBC) transfusions, and the disease transformed into AML 18 months later. The patient died 22 months after diagnosis (Pinheiro et al., 2006). This patient presented the features seen in previously reported patients: male, advanced age, severe anemia, hypercellular bone marrow, eosinophilia, basophilia, and increased micromegakaryocytes (Marisavljevic et al., 2004; Pinheiro et al., 2006). Most important, all of these cases were associated with a grim prognosis.

Therapeutic approaches to MDS/CMML should be based on the specific clinical features and risk stratification of each patient. Two new hypomethylating agents, 5-azacytidine and decitabine, have recently been approved for the treatment of MDS/CMML and are recommended by consensus guidelines for higher risk MDS patients who are ineligible for hematopoietic stem cell transplantation (NCCN, 2011).

The aim of this report is to present a case of CMML with normal cytogenetics at diagnosis that evolved into AML with $\mathrm{i}(17 \mathrm{q})$ shortly after stopping treatment with decitabine (with significant response). We also review literature reports of AML with myelodysplasiarelated changes with $\mathrm{i}(17 \mathrm{q})$. To the best of our knowledge, this report is the first of AML with myelodysplasia-related changes with $\mathrm{i}(17 \mathrm{q})$ after the use of a hypomethylating agent. 


\section{MATERIAL AND METHODS}

\section{Case report}

A 57-year-old man was diagnosed with CMML in 2007. Peripheral blood tests at presentation gave the following results: hemoglobin $=13.4 \mathrm{~g} / \mathrm{dL}$; leukocytes $=24,400 / \mu \mathrm{L}$; monocytes $=2684 / \mu \mathrm{L}$, and platelets $=54 \times 10^{9} / \mathrm{L}$. Bone marrow cytogenetics presented 46,XY[20]. According to the World Health Organization classification, CMML is considered a myelodysplastic/myeloproliferative neoplasm. Twenty-four months later, after short-term treatment with hydroxyurea, blood tests gave the following results: hemoglobin $=9.9 \mathrm{~g} / \mathrm{dL}$; leukocytes $=29,700 / \mu \mathrm{L} ;$ monocytes $=1458 / \mu \mathrm{L}$, and platelets $=12 \times 10^{9} / \mathrm{L}$. Recombinant erythropoietin was initiated with no response, and the patient became dependent on RBC and platelet transfusions. At this time, cytogenetic analysis showed 46,XY[20]. Decitabine was started at $20 \mathrm{mg} /$ $\mathrm{m}^{2}$ per day for 5 days of a 6 -week cycle. Severe febrile neutropenia developed after the third cycle, and treatment was delayed. By the fourth cycle, the patient showed progressive hematological improvement characterized by $\mathrm{RBC}$ transfusion independence and higher platelet count. The patient decided to discontinue treatment after 9 cycles. Six months later, the patient reported fatigue. The results of repeated bone marrow aspiration were compatible with AML with myelodysplasia-related changes, and cytogenetic analysis revealed 46,XY,i(17q) [20] (Figure 1). The patient died of sepsis shortly thereafter.

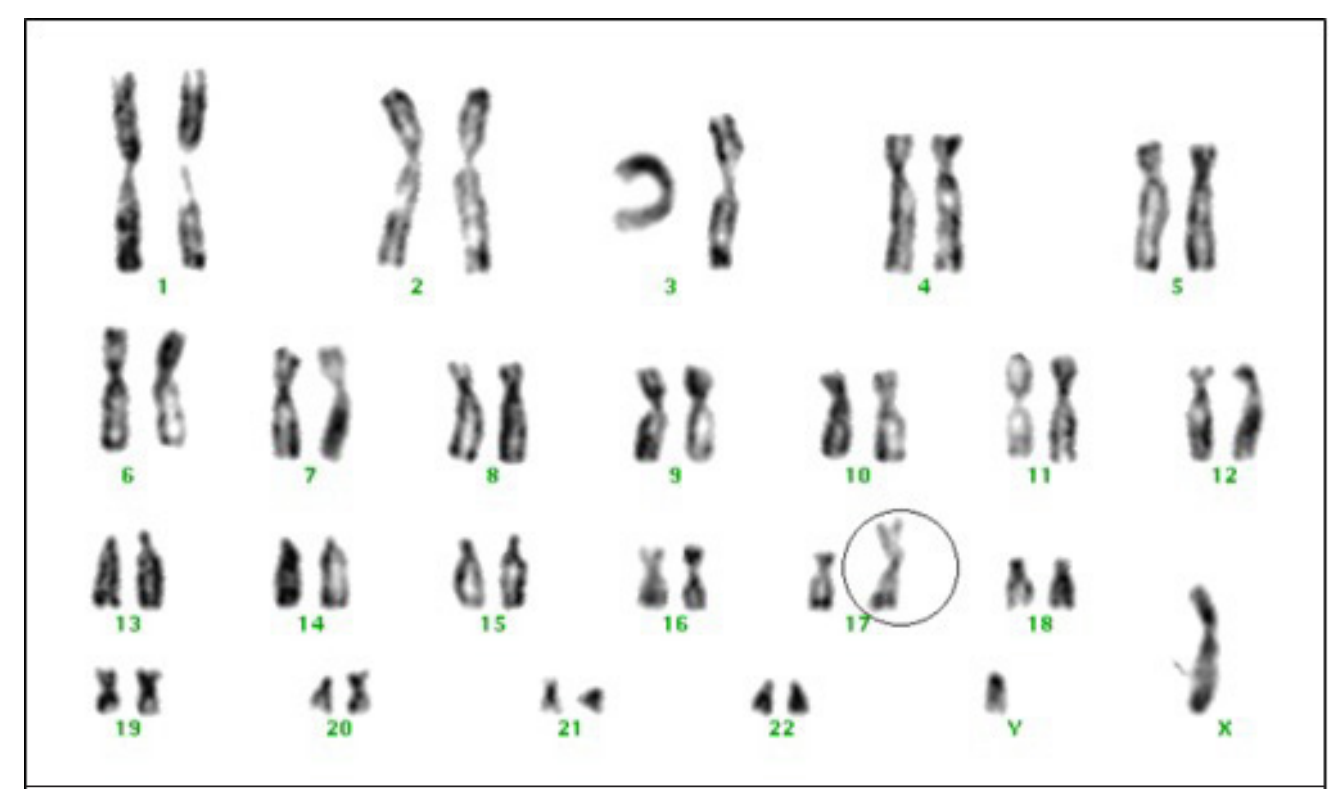

Figure 1. Karyotype from a patient with chronic myelomonocytic leukemia (CMML) treated with decitabine showing an $\mathrm{i}(17 \mathrm{q})$ shortly after stopping treatment. 


\section{RESULTS AND DISCUSSION}

$\mathrm{i}(17 \mathrm{q})$ is a marker of unfavorable prognosis in myeloid disorders (Marisavljevic et al., 2004; Pinheiro et al., 2006). It has been reported most commonly during AML transformation of typical or atypical chronic myeloid leukemia (CML). These cases always show the same features: adult patient, mixed chronic myeloproliferative-myelodysplastic features, severe hyposegmentation of neutrophil nuclei, high risk for progression to AML (64\% transformed), and median survival of 2.5 years (Marisavljevic et al., 2004; Pinheiro et al., 2006).

The case presented herein is a CMML patient who showed an excellent response to a hypomethylating agent (decitabine). The patient showed progressive hematological improvement characterized by RBC transfusion independence and higher platelet count. These changes were characterized as a complete response to decitabine during which time the patient presented a normal karyotype. Disease progression occurred in a stepwise fashion resulting from the accumulation of genetic lesions that ultimately predisposed to genomic instability. In MDS and secondary AML patients, an extensive aberrant DNA methylation involving thousands of genes is observed compared with normal CD34(+) bone marrow cells or de novo AML blasts (Shen et al., 2010). Methylation, therefore, seems to increase as disease progresses from lower to higher risk status (Shen et al., 2010). The patient reported herein showed significant hematological response to decitabine by cycle 4 and experienced relapse once therapy was stopped, corroborating the hypothesis that hypermethylation is an acting phenomenon in MDS-associated physiopathologic processes. In addition, the karyotype during transformation presented $\mathrm{i}(17 \mathrm{q})$, a marker of unfavorable prognosis.

To the best of our knowledge, this report is the first of i(17q) after the use of a hypomethylating agent for the treatment of MDS/MPN. Another very important marker of this case is the diagnosis of AML with myelodysplasia-related changes (a disorder associated with a grim prognosis).

We performed a systematic search of the medical literature for studies evaluating i(17q) in MDS/MPN between 1985 and 2011 using the PubMed database. The search was conducted using the following combination of key words: myelodysplastic or myelodysplasia and $\mathrm{i}(17 \mathrm{q})$; myeloproliferative and $\mathrm{i}(17 \mathrm{q})$; acute myeloid leukemia and $\mathrm{i}(17 \mathrm{q})$; decitabine and $\mathrm{i}(17 \mathrm{q})$, and hypomethylating therapy and $\mathrm{i}(17 \mathrm{q})$. The principal objective was to find reports of patients who presented $\mathrm{i}(17 \mathrm{q})$ during AML transformation secondary to MDS/MPN disorder. We reviewed cases of AML transformation secondary to MDS/MPN disorder with i(17q). These cases are listed in Table 1.

\begin{tabular}{|c|c|c|c|}
\hline Author, year & Disease at diagnosis/No. cases & Karyotype at AML transformation & Prognosis \\
\hline Goh and Rubins, 1985 & CML Ph- $/ 2$ cases & $i(17 q) i(17 q),+13$ & Unfavorable \\
\hline Fioretos et al., 1999 & $\mathrm{CML} / 8$ cases & $i(17 q)$ & Unfavorable \\
\hline Oren et al., 1999 & $\mathrm{CML} / 1$ case pediatric & $\mathrm{i}(17 \mathrm{q})$ & Unfavorable \\
\hline Hernández-Boluda et al., 2000 & $\mathrm{CML} / 12$ cases & $\mathrm{i}(17 \mathrm{q})$ & Unfavorable \\
\hline Mihara et al., 2007 & MPD/MDS/1 case & $\mathrm{i}(17 \mathrm{q})$ & Unfavorable \\
\hline Nishida et al., 2008 & MPD/MDS/ 1 case & $\mathrm{i}(17 \mathrm{q})$ & Unfavorable \\
\hline Park et al., 2009 & $\mathrm{MDS} / 1$ case & $\mathrm{i}(17 \mathrm{q}),+14$ & Unfavorable \\
\hline
\end{tabular}

$\mathrm{CML}=$ chronic myeloid leukemia; $\mathrm{MPD}=$ myeloproliferative disorder. 
Goh and Rubins (1985) have reported an isochromosome of the long arm of chromosome 17 in 2 patients diagnosed initially with Philadelphia-negative CML. At the time of AML transformation, one of the patients presented trisomy 13 in addition to $i(17 q)$. Both patients had a grim prognosis. Fioretos et al. (1999) described 21 cases of $\mathrm{i}(17 \mathrm{q})$ among myeloid disorders, but only 8 were AML secondary to CML in blast crisis. The cases were associated with short survival. Oren et al. (1999) have reported a case of acute promyelocytic leukemia with $\mathrm{i}(17 \mathrm{q})$ after BCR/ABL-positive CML. The case was a pediatric patient with $\mathrm{i}(17 \mathrm{q})$ after 3.5 years of treatment for BCR/ABL-positive CML. Hernández-Boluda et al. (2000) have described 12 cases of $\mathrm{i}(17 \mathrm{q})$ during blast crisis in Philadelphia-positive CML. All of these patients presented features of myeloproliferative disorder with short survival of only 22 months, and all of these cases are of AML transformation secondary to CML with unfavorable prognosis. The case we report herein is the first of AML transformation with $\mathrm{i}(17 \mathrm{q})$ secondary to CMML, a clonal hematopoietic neoplasia characterized by persistent peripheral blood monocytosis $\left(>1.000 / \mathrm{mm}^{3}\right)$ and no Philadelphia or BCR-ABL1 fusion. Of utmost importance, the patient in our current report presented $\mathrm{i}(17 \mathrm{q})$ only after stopping the hypomethylating agent.

Mihara et al. (2007) have described a 69-year-old man with AML and an isolated i(17q) karyotypic abnormality who transformed from MPD/MDS. The patient was treated with anti-leukemic drugs including anthracyclines that controlled the disease for only 6 months. Nishida et al. (2008) have reported a similar case of $\mathrm{i}(17 \mathrm{q})$ in a patient with leukemic transformation from MDS/MPD.

Park et al. (2009) have recently described a case of concomitant i(17q) and trisomy 14 in a patient with MDS in leukemic transformation. The patient was a 66-year-old Korean man with very poor prognosis. Kim et al. (2010) have also shown a case of i(17q) associated with other abnormalities. The patient had acute promyelocytic leukemia with $t(15 ; 17)$ (q22;q21) rearrangement combined with ider(17), but notably, this case was not one of AML with myelodysplasia-related changes.

We report the first case of $i(17 q)$ after the use of a hypomethylating agent for the treatment of MDS/MPN. Decitabine (5-aza-2'-deoxycytidine) is a cytosine nucleoside analog that, once incorporated into DNA, acts as a DNA methyltransferase inhibitor, reversing aberrant hypermethylation known to silence a variety of genes, including tumor suppressor genes (Shen et al., 2010). We do not know the real prognosis of i(17q) after stopping these drugs, but we speculate that it is as grim as that for patients who transform from CML. New reports are necessary to confirm this finding.

\section{ACKNOWLEDGMENTS}

Research supported by CAPES, CNPq, and FUNCAP.

\section{REFERENCES}

Brunning R, Orazi A, Germing U and LeBeau MM (2008). Myelodysplastic Syndromes/Neoplasms, Overview. In: WHO Classification of Tumours of Haematopoietic and Lymphoid Tissues (Swerdlow SH, Campo E, Harris NL, Jaffe ES, et al., eds.). 4th edn. IARC Press, Lyon, 88-93.

Fioretos T, Strömbeck B, Sandberg T and Johansson B (1999). Isochromosome 17q in blast crisis of chronic myeloid leukemia and in other hematologic malignancies is the result of clustered breakpoints in $17 \mathrm{p} 11$ and is not associated with coding TP53 mutations. Blood 94: 225-232.

Goh K and Rubins J (1985). Isochromosome 17q in Ph-negative chronic myelocytic leukemia. Cancer Genet. Cytogenet. 
14: $323-330$

Hernández-Boluda JC, Cervantes F, Costa D, Carrio A, et al. (2000). Blast crisis of Ph-positive chronic myeloid leukemia with isochromosome 17q: report of 12 cases and review of the literature. Leuk. Lymphoma 38: 83-90.

Kim M, Lee SA, Park HI, Oh EJ, et al. (2010). Two distinct clonal populations in acute promyelocytic leukemia, one involving chromosome 17 and the other involving an isochromosome 17. Cancer Genet. Cytogenet. 197: 185-188.

Marisavljevic D, Rolovic Z, Panitic M, Novak A, et al. (2004). Chromosome 17 abnormalities in patients with primary myelodysplastic syndrome: incidence and biologic significance. Srp. Arh. Celok. Lek. 132: 10-13.

Mihara K, Kido M, Nakaju N, Fukumoto S, et al. (2007). Exacerbation of acute leukemia bearing isolated i(17q) along with proliferation of blasts with high BMI-1 expression. Rinsho Ketsueki 48: 659-663.

Mitelman (1995). Catalog of Chromosome Aberrations in Cancer. 5th edn. Available at [http://cgap.nci.nih.gov/ Chromosomes/Mitelman]. Wiley-Liss, New York. Accessed September 14, 2012.

NCCN (2011). National Comprehensive Cancer Network. Clinical Practice Guidelines in Oncology - v.2. 2011. Myelodysplastic Syndromes. Available at [http://www.nccn.org]. Accessed March, 2011.

Nishida H, Ueno H, Park JW and Yano T (2008). Isochromosome i(17q) as a sole cytogenetic abnormality in a case of leukemic transformation from myelodysplastic syndrome (MDS)/myeloproliferative diseases (MPD). Leuk. Res. 32: 1325-1327.

Oren H, Düzovali O, Yüksel E and Sakizli M (1999). Development of acute promyelocytic leukemia with isochromosome 17q after BCR/ABL positive chronic myeloid leukemia. Cancer Genet. Cytogenet. 109: 141-143.

Park TS, Song J, Lee JH, Kim JS, et al. (2009). Concomitant isochromosome 17q and trisomy 14 in a patient with myelodysplastic syndrome in leukemic transformation. Ann. Clin. Lab. Sci. 39: 176-181.

Pinheiro RF, Chauffaille ML and Silva MR (2006). Isochromosome 17q in MDS: a marker of a distinct entity. Cancer Genet. Cytogenet. 166: 189-190.

Shen L, Kantarjian H, Guo Y, Lin E, et al. (2010). DNA methylation predicts survival and response to therapy in patients with myelodysplastic syndromes. J. Clin. Oncol. 28: 605-613. 